\title{
Robot, Pass Me the Scissors! How Robots Can Assist Us in the Operating Room
}

\author{
Juan P. Wachs \\ Purdue University, West Lafayette, IN 47906, USA \\ jpwachs@purdue.edu
}

\begin{abstract}
The inclusion of robotics and automation to support and augment surgical performance offers the promise of shorter operating times, higher accuracy and fewer risks compared with traditional, human-only surgery. This paper discusses current research in the area of surgical robotics and human-robot collaboration. A multimodal robotic scrub nurse (Gestonurse) for the operating room (OR) is presented as a case study. Gestonurse assists the main surgeon by passing surgical instruments, thereby releasing the surgical technician to perform other tasks. Such a robotic system has the potential to reduce miscommunication and compensate for understaffing. The implications of the introduction of surgical robots, as assistants rather than autonomous agents, are discussed in terms of the societal and technological requirements. Quantitative and qualitative findings are presented as evidence to support the guidelines discussed.
\end{abstract}

Keywords: Gesture recognition, operating room, human computer interaction.

\section{$1 \quad$ Introduction}

Understaffing of nurses is a chronic problem in American hospitals that is expected to continue-a shortage of 260,000 registered nurses is (RNs) projected by 2025 [1]. Research conducted by the Robert Wood Johnson Foundation, NPR, and the Harvard School of Public Health found that the problem was not necessary nurses understaffing but nurses overworked. 34\% of patients hospitalized during 2011 thought that nurses were not available when needed. While ideally the expectation is to have one nurse for every three patients in her ER, the reality makes the nurse to care for five patients to eight. This issue points to a shortage of nursing care in hospitals and other healthcare facilities. Moreover, there is a growing concern about the nursing staff being stretched even more thinly in the coming years. This shortage has been linked to an array of negative outcomes such as higher transmission rates of antibiotic resistant strains of illness, cardiac arrest and urinary tract infections [2]. Additionally, recent studies have shown that inpatient mortality risk is nearly $6 \%$ higher in units understaffed with nurses compared to fully staffed units [3]. As an example, another study [4] found that 11 to $14 \%$ patient mortality in Pennsylvania and New Jersey was directly related to unsuitable patient-to-nurse staffing ratios and burnout symptoms compared to other states in the US. A similar picture has been found in 12 countries in Europe [37]. 
Because of this shortage, it is important to find new ways to use technological solutions for highly mechanistic tasks in health care settings. This frees nurses to focus on more complex tasks, allowing outcomes to be improved for all.

\section{Why Do We Need Robots in the OR?}

Currently, in the United States, errors in medical care delivery are the principal cause of inpatient mortality and morbidity (over 98,000 deaths annually). Research assessing verbal and non-verbal exchanges in the operating room (OR) shows that communication failures are frequent: commands are delayed, incomplete, not received at all, and frequently left unresolved. One study found that $31 \%$ of all communications in the OR represent failures, a third of which had a negative impact on the patient [5-8]. Another study found that $36 \%$ of communication errors were related to equipment use. New technologies could have a crucial impact on OR communication- Adaptable surgical robotic assistants that understand implicit and explicit communication can reduce the number of errors related to miscommunication. One of the uses of surgical robots is to augment the surgeon dexterity and physical abilities by offering a set of customizable set of skills while reducing errors intrinsic to humans [9]. Surgical assistants come in two flavors: surgeon extenders and auxiliary surgical support robots. The first type is controlled uniquely by the surgeon and can enhance the surgeon's dexterity during surgery (such as by eliminating hand's tremors). The second type works side-by-side with the surgeon and supports her task in a variety of functions, such as holding the endoscope. This type of robot can respond to joysticks, voice, touch-pads, and head trackers based interfaces. Natural verbal and nonverbal interfaces can allow surgeons to interact naturally with the robot (assistant) without requiring the surgeon learn in a new set of functions or wearing encumbering sensors. Within the social robotics community, the belief is that surgical robotic assistants, by reducing the miscommunications, will: 1) reduce morbidity and mortality risks; 2) treat of otherwise untreatable pathologies; and 3) reduce operating times. While such robotic systems in the operating room (OR) have been studied, nonverbal interaction as a means of robot interaction, has never been applied to the surgical theater.

\section{Previous Work on Surgical Robots}

Previous research on surgical extenders robots includes an assistive robot for object picking [10], haptic feedback controlled robots, such as SOFIE [11] or Agovic's [12] robot, and the commercially available da Vinci® surgical suite [13] which was developed to conduct minimally invasive and safer procedures for planned endoscopic and laparoscopic surgeries.

Robotic scrub nurses (RSN) is a type of auxiliary support robots that has some level of autonomy and cognitive capabilities. For example, Kochan et al., developed "Penelope" [14] which delivers and retrieves surgical instruments and is controlled by voice. Another voice-controlled robot developed by Carpintero et al., [15] relies on computer vision techniques to recognize, pick and return surgical instruments. 
The EASOP [16] is a robotic endoscope holder controlled by means of speech. The RSN system developed by Yoshimitsu et al., [17] used voice control for handling laparoscopic surgeries. It also used depth-based action recognition for instrument use prediction. To assess the surgeon's action automatically, 3D point estimation and tracking is performed, requiring the surgeon to wear markers. These markers can compromise sterility.

Overall, the problem with voice-only systems and that they exhibit notable performance degradation [18] in loud environments such as the OR. Anesthesia machines, respirators, drills, side conversations, and other equipment affects the recognition accuracy - in such a setting, the surgeon may say "50,000 units" and the anesthetist may hear "15,000 units" [19]. Such errors could have catastrophic consequences for the patient.

Robots were developed by the military to reduce the fighters' exposure to unfriendly fire. These robots are capable to: (1) safely extract wounded from the battlefield; (2) diagnose and treat life threatening injuries within minutes; and (3) conduct surgery. The BEAR robot (The Battlefield Extraction-Assist Robot) [20] can extract wounded soldiers from the battleground. This robot is controlled remotely and understands speech commands. SRI's M7 surgical robot [21] is the first of its kind applied to a perform a tele-surgery (networked over 1,200 miles undersea) to simulate the conditions of outer space. In light of its success, it was integrated into an autonomous ultrasound-guided medical procedure under similar conditions. Trauma Pod [22] is a glimpse to the next generation of mobile robotic surgery platforms capable of conducting life-saving procedures in hostile environments. It is controlled through speech messages. A surgical deployed robot that responds to speech commands to deliver surgical tools retrieves them as soon as they are not longer needed [23] was developed by Treat et al. The instruments were recognized through computer vision, and a cognitive architecture was used for decision making. None of the robots discussed so far address the problem of multimodal interaction between surgeon and robot.

\section{Gestonurse: A Robotic Assistant That Understands Verbal and Non-verbal Communication}

A real-time RSN (Fig. 2) capable to passing surgical instruments to the surgeon when requested by speech and/or gesture has been developed by Jacob et al., [24]. This is the first time that non-verbal cues (hand gestures) were considered as a modality of interaction with surgical robots in the OR. Using gesture interaction has the advantage that it does not require surgeon total re-training and it is a totally sterile mean of control. Hand gestures are currently being used by surgeons to request the instruments as part of the OR technique [25-27] for surgery. Another advantage is that non-verbal communication is not affected by ambient noise in the OR.

Gestural commands are recognized from depth images acquired from a commodity camera (Kinect) and speech requests are gathered from a microphone. The FANUC robotic arm hands the required instrument to the surgeon (see Figure 2), as soon as the commands were recognized. Gestures were also used to switch between states in the RSN's state machine. 
The system architecture of the robotic system is presented in Figure 3. The depth maps obtained from the Kinect sensor were processed by the gesture recognition module and in parallel the speech commands were captured by a microphone. These commands were interpreted using the speech recognition module. Once recognized, the command was sent through the network to the FANUC robotic arm using a Telnet interface. The robot, in turn, delivers the required surgical instrument to the surgeon and stays 'on hold' until the next command is received.

The system architecture, the computing and physical modules are briefly described in the following subsections.
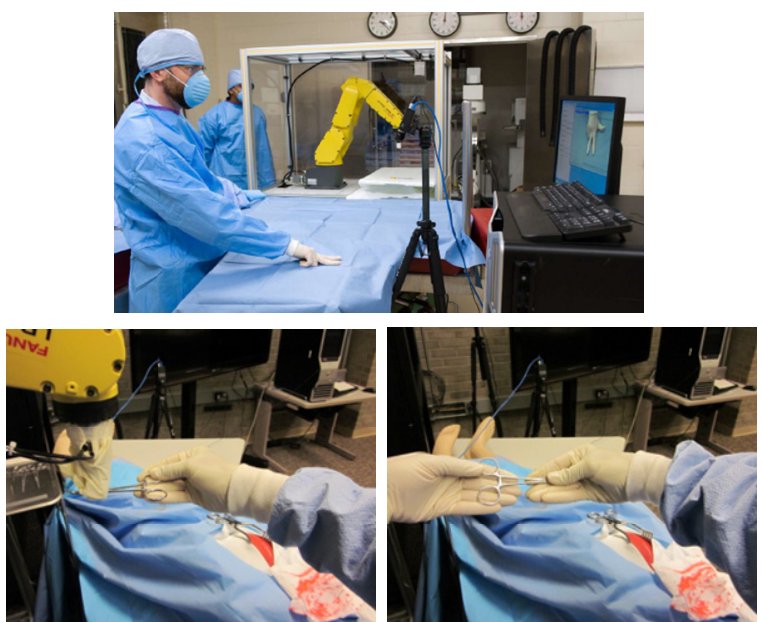

Fig. 1. (a). A prototype of the real-time RSN running at an OR (b) A sterile robot handing a surgical instrument (c) A nurse handing a surgical instrument

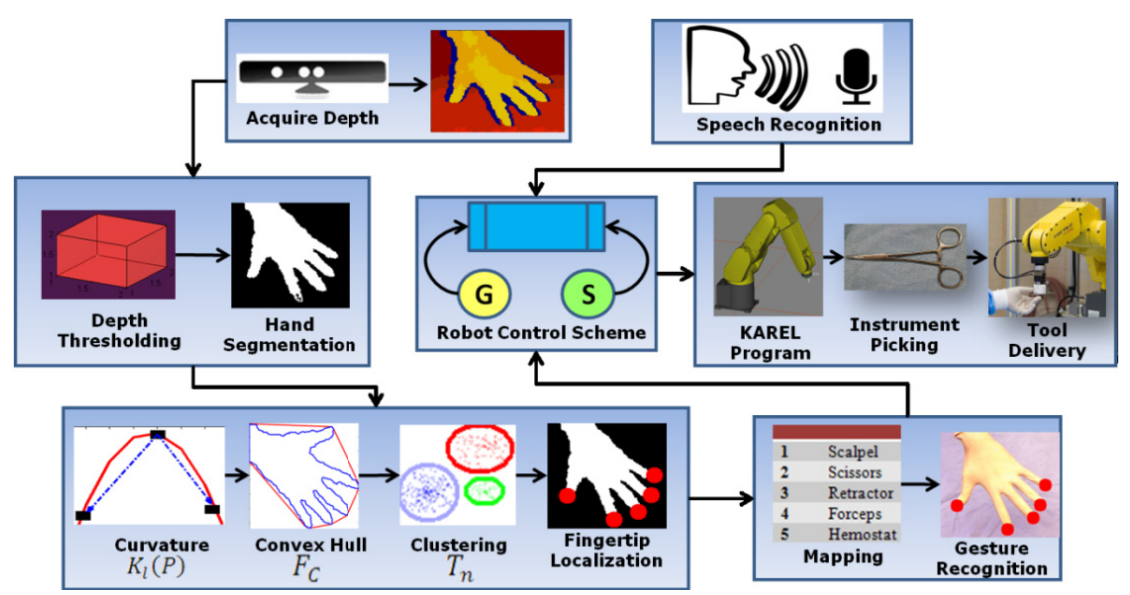

Fig. 2. System Overview 


\subsection{Pose Segmentation}

Color and depth information were used for hand segmentation and compared to assess the strength of each method. Using color cues for segmentation required finding a background model of the scene and creating hand color model. Segmentation masks from the color models were added and morphologically processed so the largest blob in the image is selected. This region represents the mask of the hand once the noise was filtered out.

When using the depth cues, the segmentation involved extracting the user's hand from the scene using the depth map obtained from the Kinect sensor. Applying a threshold operation to the depth map and selecting the region closest to the camera resulted in the mask of the hand.

The contour and convex hull were found from the images including the hand masks. This information was passed to the fingertip localization module. Finally, the two methods of segmentation were compared and the one delivering the best results was selected (the depth-based method) as the main segmentation approach.

\subsection{Fingertip Localization}

To find the fingertip, the curvature value at each point on the contour of the hand mask was computed at multiple scales. For a point on the contour to be considered a fingertip candidate, it had to be the local minimum over several scales and its curvature lower than a given threshold. The set of candidate points included all those points that met the previous criteria after contour discretization, and those points affected by the presence of noisy segmentation. This set was reduced by discarding points too far from the convex hull and clustering the resulting points together. The true fingertip was represented by the centroid of each cluster.

\subsection{Hand Gesture Recognition}

The hand pose was recognized after the fingertips were detected and located within the hand. The number of fingertips in the image was one-to-one matched to the surgical instruments. Once the required instrument was detected from the image, this request was sent to the state machine.

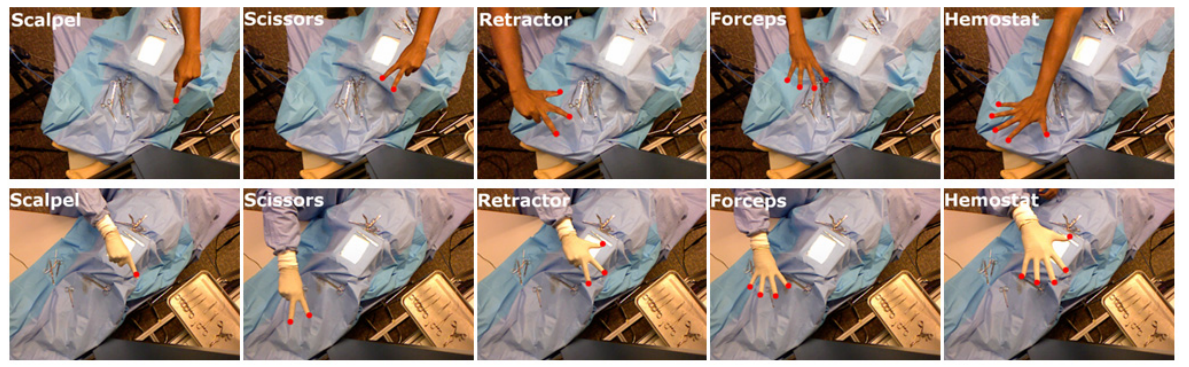

Fig. 3. Samples from the use-case with detected fingertips marked with red circles 


\subsection{Speech Recognition}

The speech recognition module was responsible of recognizing each spoken command (from the surgeon) and sending the instrument request to the state machine for further processing. A fixed steered microphone was oriented towards the surgeon and it gathered acoustic information. The speech commands were recognized using the open source CMU Sphinx [28] toolkit.

\subsection{State Machine}

The state machine was responsible for processing requests coming from the gesture and speech recognition modules, and it was meant to deliver instruments, put the system 'on hold' (sleep mode), switch the system to "active" mode, or disable the speech recognition module. Disabling the speech modality was necessary in scenarios where the ambient noise was too loud which can result in false triggers. The gesture and speech recognition modules operate in parallel.

\subsection{Instrument Pick and Delivery}

A six degrees of freedom (DOF) FANUC robot was programmed using a combination of programming platforms, including: KAREL, a FANUC proprietary language used to control the robots and teach-pendant (TP) programs. The position of the surgical instruments was hard-coded and the corresponding coordinates were stored as part of the TP programs. The programs controlling the FANUC robot were each triggered when a request (gestural or verbal) was detected over the telnet interface. The robot proceeded to pick the instrument from its pre-programmed position using a latexencapsulated magnetic gripper and passed it to the surgeon. When the instrument was delivered, the arm would return to its 'HOME' position and switch to the "wait" mode where it awaits a request for a new surgical instrument.

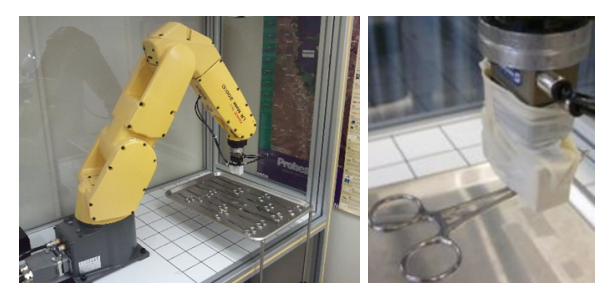

Fig. 4. (a) FANUC Robot (b) Latex-encased magnetic gripper

\subsection{Human Robot Collaboration in a Mock Surgery}

Experiments involving human-robot collaboration were performed using a patient's phantom simulator (Fig. 5). The performance of the system was assessed using common surgical task -- an abdominal incision and closure. The purpose of this surgical exercise was to penetrate the peritoneal cavity without puncturing an inflated balloon, manually examining the surroundings of the cavity, and final closing the abdominal wall without bursting the balloon. The task required the proper handing and use of 
surgical instruments such as scalpel and forceps Two types of subjects were involved in the experiment, the surgeon and an assistant, and two scenarios were studied: with robotic assistant to complete the task, and standard collaboration between surgeon and nurse without the robot. The economy of movements, learning times and recognition accuracy were assessed by comparing the performance of the mock surgical procedure under the two experimental conditions. It was found that that the robotic assistant improved the surgical procedure by reducing the number of movements (lower variance in the picking position) [29]. Robust recognition accuracy (97\%) and short completion times after training ( 250 seconds) were also reported by these studies $[30,31]$.

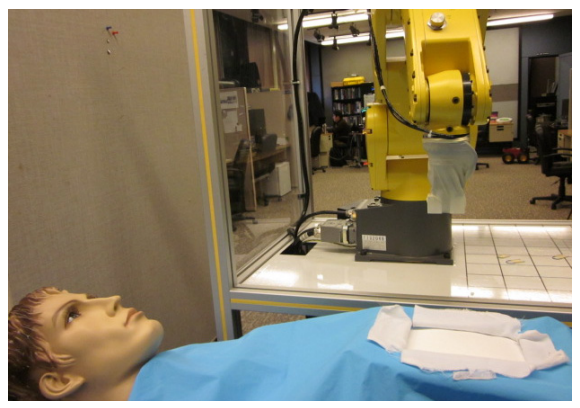

Fig. 5. A surgical simulator
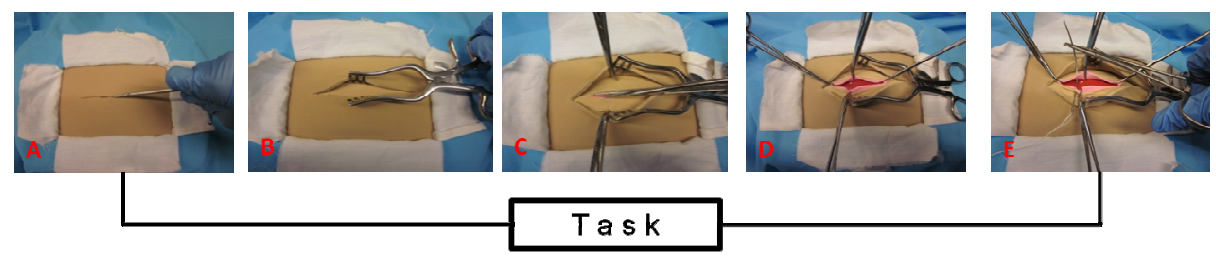

Fig. 6. Different stages of the mock abdominal incision. Stage (A). Incision; (B) Exposure of linea alba; (C) Enlarge the incision using scissors; (D) Incise the linea alba; (E) Close the incision.

\section{Perception and Trust}

While technically robots could complement/supplement personnel in hospitals, by working side by side with caregivers and patients, the robots need to be accepted socially by the hospital's staff. Robots need to be perceived as team-mates rather than threatening agents which purpose is taking away jobs. They need to build trust among the co-workers and patients, rapport must be built and responsibilities need to be shared.

\subsection{Perception}

Social acceptance is a fundamental key challenge for successful adoption of robots in hospitals. The main reason for this is that robots could replace and/or support highly 
educated workers in surgery with robot partners. Only a small number of studies have focus on the perception of robots as team co-workers in the OR. Wasen [32] looked at teamwork and human-robot interaction in surgery through ethnographic research tools to study social understanding and acceptance of surgical robots during a two-and-ahalf-year study (2003-2005) involving four hospitals, one in the United States and three in Scandinavia. The case studies observed included coronary by-pass and prostate removal surgeries performed by surgeons with robotic assistance (AESOP, ZEUS and da-Vinci). The way that the AESOP system is designed implies that one surgical nurse is replaced by the robot which does the same job as the human assistant. Qualitative results of this study showed that surgeons were eager to accept surgical robots because "the robot's functionality provided them with additional support and increased their independence in the work place." [32]. From the surgical assistants stand point, the robotic assistant forced the assistants to participate in a very different way in the procedure due to the fact that the lead surgeon was in control of almost all the aspects related to the surgery. Anecdotic evidence shows that surgical nurses are more reluctant about the introduction of surgical robots to the OR due to the fact that they present a potential threat to their job stability.

\section{$5.2 \quad$ Trust}

Trust can affect the hospital's staff willingness to share existing surgical tasks, provide support to the robot [33], and provide information to enhance its performance. In healthcare contexts, a caregiver must trust that a robotic co-worker will protect the interests and welfare of the patients and to ensure the procedures' success with minimum risks. At the same time, the caregiver needs to trust that the robotic assistant will not "displace" the teammate from the task in hand. Trust allocation can have serious implications in the outcomes of the care, e.g. too much trust is placed on the robot can lead to physical risks to the patients - increasing mortality and morbidity risks (misuse) [34]. If too little trust is placed in the robot (disuse), the team may stop using the system altogether. An important observation is that trust decreases with more complex and cognitive demanding tasks, such as surgery, because the chances of error occurrences are higher and the costs (social and economic) of those errors are enormous; and therefore the team mate is more likely to rely on herself [35].

Even though trust assessment through objective measures is difficult to accomplish (mostly since the reported values are subjective), a number of factors have been suggested to estimate proxies for trust levels. Some of these factors include system usability, proximity of the robot, proxemics, situation awareness, and characteristics of the task and the environment [36]. A special emphasis should be given to ethnographic studies involving surgical nurses to clarify what are the issues that are concerning them the most. Findings can be used to create enhanced human-robot team models of trust, and help determine ways that mistrust may be mitigated, while assuring that there is a backup plan given that the system was over trusted.

\section{Challenges and Recommendations}

The entire human (perception, trust) and machine (accuracy) related characteristics may have implications for the future adoption of surgical robots as assistants in healthcare 
environment. Following specific recommendations and trying to address the existing challenges may reduce initial perception barriers and foster trust development by giving the user the option to participate in the implementation and integration of the robots in the OR. The surgical staff will be pivotal on the gathering of information about robot capabilities, system behaviors, and potential risks. In addition, a simple way to enhance the staff's perception about the robot is through fundamental training on how the tasks can be best shared among the participants, without fears but offering a fault-safe strategy to backup system errors. Based on previous findings, the following list of requirements was identified. This is not a comprehensive list, but it highlights the most common requirements that the surgical staff pointed out:

1) Suitable dexterity: Manipulation of surgical equipment requires a high level of dexterity (the hand has $27 \mathrm{DOF}$ ). For the most part, the surgical nurse needs to hand over the instrument to the surgeon, and pick them from the surgical site. A robotic arm with seven DOF and a gripper similar to tweezers may complete the task as good as a person. Nevertheless, more complex tasks such as opening a suture bag, or applying suction catheter may require more complex robots (e.g. dual arms) with more dexterous hands.

2) Multimodality: To successfully recognize the subtleties of communication that occur between humans, gestures, body language, gaze and speech need to be recognized. When more than one modality is used, the robot must be capable to resolve ambiguity (different requests expressed by different modalities).

3) Fast response: The action carried out by the robotic assistant must be promptly without delays due to actuators or due to processing requests. The immediate response should be as quick as an experienced assistant standing in the close proximity to the surgeon. Excessive speed is not advisable since it may come on the account of precision, or exhibit jerky movements.

4) Prediction (mind readers): Experienced nurses are also called mind readers because their ability to anticipate the surgeon's need. A robotic assistant must have robust inference and prediction mechanisms to perform the activity which is has been assigned to complete in support of the surgeon. Given that the prediction is not correct, ways to mitigate the mistakes and their consequences need to be included in the system.

5) Predictable: The surgical team will expect to see the robotic assistant to respond in the same fashion to similar requests under the same conditions every time. Unexpected behavior has the potential to distract the surgical team, create delays and even increase the risks of physical contact with the robot.

6) Accuracy and Precision: The surgeon's commands/requests must be interpreted accurately. A true hit rate over $99 \%$ with almost no false alarms is required to perform as good as a human assistant. This performance should be attainable in complex environment with variable light changes unfixed settings. Precise movements are also necessary to assure the grasp of cluttered small objects, like surgical equipment, sponges and gauzes.

7) Safety: Operator safety in industrial robotics has been addressed by defining a physical area (safety envelope) that is monitored for intrusions. This is not applicable for collaborative tasks in close human-robot interaction where physical contact is required. To avoid injuries due to collisions with sharp instruments, impact forces need to be reduced through effective design, or avoiding the collisions altogether through strategies for obstacle avoidance. 
Based on this research, it is expected that some of these requirements and challenges will be addressed to allow the surgical team and robotic assistants to support intrinsically the development of effective, safe and more cost efficient human robot collaboration in the operating theater.

\section{Conclusion}

The shortage of nursing care in hospitals and other health care facilities has been linked to an array of negative outcomes such as higher transmission rates of antibiotic resistant strains of illness, cardiac arrest and urinary tract infections [5]. Inpatient mortality risk is nearly $6 \%$ higher in units understaffed with nurses compared to fully staffed units [7-8]. New technologies could have a crucial impact on OR communication- Adaptable surgical robotic assistants that understand implicit and explicit communication can reduce the number of errors related to miscommunication and make up for the lack of employed nurses.

The current research described a number of surgical robots designed to support the surgical team by being a co-worker rather than a tele-operated device. A robotic scrub nurse - Gestonurse - was presented as a case study of the existing challenges and limitations of robots in the OR. The system discussed, showed the feasibility for the implementation of a robot capable to understand non-verbal communication (hand gestures) and speech commands with a recognition accuracy of over $97 \%$. Gestonurse has been validated in a mock surgery - an abdominal incision and closure. Results on economy of movements showed that the robotic assistant improved the surgical procedure by reducing the number of movements which is closely related to the concept of economy of movements in the operating room. Improving the effectiveness of the operating room can potentially enhance surgical outcomes without affecting the performance time.

In the last section of this work, it was described the requirements of surgical robotic assistants in terms of the technical and societal needs in today's operating room. While some of these requirements vary depending on the scope of the robot; there is a common theme- the need to be socially accepted and technically skilled as their team mates. Implications related to the introduction of surgical robots in the surgical setting, as assistants rather than autonomous agents, will have sociological and technological effects that are likely transform healthcare as we know it today. Scientific awareness of presented challenges to be addressed will guide the next generation of robots so they will play a central role in this transformation.

\section{References}

1. Buerhaus, P.I., Auerbach, D.I., Staiger, D.O.: The Recent Surge In Nurse Employment: Causes And Implications. Health Affairs 28(4), 657-668 (2009)

2. The Effect of Health Care Working Conditions on Patient Safety, Agency for Healthcare Research and Quality, Rockville, MD, Summary, Evidence Report/Technology Assessment: Number 74 AHRQ Publication No. 03-E024 (2003)

3. Needleman, D.J., Buerhaus, P., Pankratz, V.S., Leibson, C.L., et al.: Nurse staffing and inpatient hospital mortality. New England Journal of Medicine 364(11), 1037-1045 (2011) 
4. McHugh, M.D., Brooks Carthon, M., Wu, E., Kelly, L., Sloane, D., Aiken, L.H.: Impact of nurse staffing mandates on safety-net hospitals: Lessons from California. The Milbank Quarterly 90, 160-186 (2012)

5. Firth-Cozens, J.: Why communication fails in the operating room. Qual. Saf. Health Care 13(5), 327 (2004)

6. Lingard, L., Espin, S., Whyte, S., et al.: Communication failures in the operating room: an observational classification of recurrent types and effects. Qual. Saf. Health Care 13, 330334 (2004)

7. Healey, A.N., Undre, S., Vincent, C.A.: Defining the technical skills of teamwork in surgery. Qual. Saf. Health Care 15, 231-234 (2006)

8. Carthey, J., de Laval, M.R., Wright, D.J., et al.: Behavioural markers of surgical excellence. Safety Science 41, 409-425 (2003)

9. Taylor, R.H., Stoianovici, D.: Medical Robotics in Computer-Integrated Surgery. IEEE Transactions on Robotics and Automation 19(5) (2003)

10. Borenstein, J., Koren, Y.: A mobile platform for nursing robots. IEEE Transactions on Industrial Electronics (2), 158-165 (2007)

11. Bedem, V.L.: Realization of a demonstrator slave for robotic minimally invasive surgery. Ph.D. dissertation, Technische Universiteit Eindhoven (2010)

12. Agovic, A., Levine, S., Papanikolopoulos, N., Tewfik, A. (2010) Haptic interface design considerations for scrub nurse robots in microsurgery. 18th Mediterranean Conference on Control \& Automation (MED), 2010 , pp.1573-1578.

13. Intuitive Surgical, da Vinci Surgical System, Online

14. Kochan, A.: Scalpel please, robot: Penelope's debut in the operating room. Industrial Robot: An International Journal 32(6), 449-451 (2005)

15. Carpintero, E., Perez, C., Morales, R., et al.: Development of a robotic scrub nurse for the operating theatre. In: 2010 3rd IEEE RAS and EMBS International Conference on Biomedical Robotics and Biomechatronics (BioRob), pp. 504-509 (2010)

16. Nathan, C., Chakradeo, V., Malhotra, K., D’Agostino, H., Patwardhan, R.: The VoiceControlled Robotic Assist Scope Holder AESOP for the Endoscopic Approach to the Sella. Skull Base 12, 123-132 (2006)

17. Yoshimitsu, K., Miyawaki, F., Sadahiro, T., et al.: Development and evaluation of the second version of scrub nurse robot (SNR) for endoscopic and laparoscopic surgery. In: Intelligent Robots and Systems, IROS 2007, pp. 2288-2294 (2007)

18. McCulloch, P., Mishra, A., Handa, A., Dale, T., Hirst, G., Catchpole, K.: The effects of aviation-style non-technical skills training on technical performance and outcome in the operating theatre. British Medical Journal 18(2), 109 (2009)

19. Mitchell, L., Flin, R.: Non-technical skills of the operating theatre scrub nurse: literature review. Journal of Advanced Nursing 63(1), 15-24 (2009)

20. Purdy, E.: The Increasing Role of Robots in National Security. In: Greig, J.M. (ed.) Defense AT\&L, vol. XXXVII (2008)

21. King, H., Low, T., Hufford, K., Broderick, T.: Acceleration compensation for vehicle based telesurgery on earth or in space. In: The International Conference on Intelligent Robots and Systems, IROS IEEE/RSJ 2008, pp. 1459-1464 (2008)

22. Garcia, P., Rosen, J., Kapoor, C., Noakes, M., Elbert, G., Treat, M., Ganous, T., Hanson, M., Manak, J., Hasser, C., Rohler, D., Satava, R.: Trauma pod: A semi-automated telerobotic surgical system. International Journal of Medical Robotics and Computer Assisted Surgery 5(2), 136-146 (2009)

23. Treat, M.R., Amory, S.E., Downey, P.E., Taliaferro, D.A.: Initial clinical experience with a partly autonomous robotic surgical instrument server. Surg. Endoscopy 20 (2006) 
24. Jacob, M.G., Li, Y., Wachs, J.P.: A Gesture Driven Robotic Scrub Nurse. In: Proceedings of the 2011 IEEE International Conference on Systems, Man, and Cybernetics, Anchorage, Alaska, October 9-12 (2001)

25. Fulchiero, G.J., Vujevich, J.J., Goldberg, L.H.: Nonverbal hand signals: a tool for increasing patient comfort during dermatologic surgery. Dermatol Surg. 35(5), 856-857 (2009)

26. Phillips, N., Berry, E., Kohn, M.: Berry \& Kohn's operating room technique. Mosby Inc. (2004)

27. Pezzella, A.: Hand Signals in Surgery. AORN Journal 63(4), 769 (1996)

28. CMU Sphinx: Open source toolkit for speech recognition. Carnegie Mellon University (2011)

29. Wachs, J.P., Jacob, M., Li, Y., Akingba, G.: Does a robotic scrub nurse improve economy of movements? In: SPIE Medical Imaging, Image-Guided Procedures, Robotic Interventions, and Modeling Conference, San Diego, California USA, February 4-9 (2012)

30. Jacob, M., Li, Y.T., Wachs, J.P.: Gestonurse: A Multimodal Robotic Scrub Nurse. In: Proc. of the7th ACM/IEEE Intl Conf. on Human Robot Interaction (HRI 2012), Boston, Massachusetts, March 5-8 (2011)

31. Jacob, M., Li, Y.T., Akingba, G., Wachs, J.P.: 'Gestonurse' a robotic surgical nurse for handling surgical instruments in the operating room. Journal of Robotic Surgery, 1-11 (2011) ISSN: 1863-2483

32. Wasen, K.: Replacement of Highly Educated Surgical Assistants by Robot Technology in Working Life: Paradigm Shift in the Service Sector. I. J. Social Robotics 2(4), 431-438 (2010)

33. Freedy, A., DeVisser, E., Weltman, G., Coeyman, N.: Measurement of trust in humanrobot collaboration. In: Proc. Int. Symp. Collaborative Technologies and Syst., Orlando, FL, pp. 106-114 (2007)

34. Parasuraman, R., Riley, V.: Humans and automation: Use, misuse, disuse, abuse. Human Factors 39(2), 230-253 (1997)

35. Adams, B.D., Bruyn, L.E., Houde, S., Angelopoulos, P.: Trust in automated systems literature review. Defense Research and Development Canada Toronto No. CR-2003-096 (2003)

36. Desai, M., Stubbs, K., Steinfeld, A., Yanco, H.: Creating trustworthy robots: Lessons and inspirations from automated systems. In: Proc. Artificial Intelligence and Simulation of Behaviour Conv.: New Frontiers in Human-Robot Interaction, Scotland (2009)

37. Aiken, L.H., Sermeus, W., Van den Heede, K., Sloane, D.M., et al.: Patient safety, satisfaction, and quality of hospital care: Cross-sectional surveys of nurses and patients in 12 countries in Europe and the United States. British Medical Journal (2012) 